\title{
Heat Diffusion-Induced Gradient Energy Level in Multishell Bisulfides for Highly Efficient Photocatalytic Hydrogen Production
}

Bilin Wang, Shaohui Guo, Xu Xin, Youzi Zhang, Yijin Wang, Chenghua Li, Yaru Song,

Dongshan Deng, Xuanhua Li, ${ }^{*}$ Ana Jorge Sobrido, and Maria-Magdalena Titirici

Insufficient light absorption and low carrier separation/transfer efficiency constitute two key issues that hinder the development of efficient photocatalytic hydrogen production. Here, multishell $\mathrm{ZnS} / \mathrm{CoS}_{2}$ bisulfide microspheres with gradient distribution of $\mathrm{Zn}$ based on the heat diffusion theory are designed. The $\mathrm{Zn}$ distribution can be adjusted by regulating the heating rate and manipulating the diffusion coefficients of the different elements conforming the multishell photocatalyst. Because of the unique structure, a gradient energy level is created from the core to the exterior of the multishell microspheres, which effectively facilitates the exciton separation and electron transfer. In addition, stronger light absorption and larger specific surface area have been achieved in the multishell $\mathrm{ZnS} / \mathrm{CoS}_{2}$ photocatalysts. As a result, the multishell $\mathrm{ZnS} / \mathrm{CoS}_{2}$ microspheres with gradient distribution of $\mathrm{Zn}$ exhibit a remarkable hydrogen production rate of $8001 \mu \mathrm{mol} \mathrm{g-1} \mathrm{h-1,} \mathrm{which} \mathrm{is} 3.5$ times higher than that of the normal multishell $\mathrm{ZnS} / \mathrm{CoS}_{2}$ particles with well-distributed $\mathrm{Zn}$ and 11.3 times higher than that of the mixed nonshell $\mathrm{ZnS}$ and $\mathrm{CoS}_{2}$ particles. This work demonstrates for the first time that controlling the diffusion rate of the different elements in the semiconductor is an effective route to simultaneously regulate morphology and structure to design highly efficient photocatalysts.

\section{Introduction}

Hydrogen production via photocatalytic water splitting is known to be one of the most prospective and effective methods to battle the current global energy and environmental crisis. [1-8] However, to date, the best performing photocatalysts still suffer from relatively low solar-to-hydrogen conversion, to levels that lie far below the demand for practical applications.[6,8-11] The low efficiency of photocatalytic water splitting is attributed to three main reasons: i) the low utilization of sunlight, ii) the poor efficiency of 
separation and transfer of photogenerated carriers, and iii) sluggish kinetics of the hydrogen evolution reaction.[8,9,12-16] Thereby, the design and construction of a photocatalyst to broaden the visible light harvesting and accelerate separation/transfer efficiency of charges and reaction kinetics for water reduction are highly desirable.

The activity of a photocatalyst is greatly dependent on the design methodology. A rational design can lead to a photocatalyst that exhibits efficient light absorption, facilitates charge separation and transport, and accelerates surface reactions.[17-21] Morphology design and composition control are the two main strategies employed for catalyst design. So far, a favorable library of morphological structures has been devised in semiconductor photocatalysts, containing OD quantum dots,[22,23] 1D nanowires/nanorods,[8,24,25] 2D nanosheets, $[1,12,13]$ 3D hollow nanostructures,[21,26] and heterostructures. $[5,6,17]$ Among them, precisely designed hollow multishell structures possess attractive characteristics for photocatalysis, including efficient light harvesting, improved charge transfer, and high specific surface area.[17,21,27-32] Single/multishell hollow spheres,[18,33,34] hollow cubes,[35-37] egg yolk shells,[27,36,38] and other shaped hollow spheres have been reported.[19,28,39-41] In general, the preparation and synthesis of multishell structured photocatalysts can be divided into three methods according to the template used: hard templating, soft templating, and self-templating method.[17,21,26,30,34,40,42-44] Hard and soft templating methods generally use rigid materials and micelles as templates. However, these methods usually suffer from complex template removing procedures, nonuniform coatings of particles, and difficulty in selecting templates that are compatible with the targeted material.[32-34,42,45] In self-templating preparation strategies, amorphous coordination polymers can be used as synthetic precursors to produce highly complex metal oxide shells by adjusting the composition, offering a clear advantage compared to other templating methods. [18,21,33,46]

The complex multishell hollow materials with heterogeneous structures are expected to provide unique properties and functions because they retain the advantages of a single component while offering synergetic effects between the different components.[28,29,35,43,47] However, the major synthetic obstacle comes from the difficulty in accurately controlling the formation of multiple shells at the nanoscale. $[35,48]$ The precise synthesis of multishell nanostructures with two different compounds has remained yet an experimental challenge.

Attention should be also paid to the chemical composition of photocatalysts. [21,26,49] By controlling the chemical composition of the catalyst, the energy band structure can be finely tuned to promote charge separation and transfer. $[26,30,31,49,50]$ Numerous efforts have been dedicated to achieving energy level regulation through doping,[51-54] surface treatment,[55,56] constructing defect,[49,51] and designing heterojunctions, [57,58] and homojunctions.[50,56] Among them, building a gradient energy level inside the photocatalyst constitutes a very efficient way to achieve higher exciton separation efficiency.[24,50,54,56,59] However, current methods require the addition of external dopants, which can lead to the creation of recombination centers, hence decreasing the carrier mobility and lifetime.[51,52] Therefore, a new method that can produce a gradient energy level by just adjusting the elemental composition of the photocatalyst without the addition of external components would be highly desirable. 
Here, by simultaneously considering morphology control and energy band structure design, we take advantage of their cooperative effects to design a multishell $\mathrm{ZnS} / \mathrm{CoS}_{2}$ bisulfide photocatalyst with gradient distribution of $\mathrm{Zn}$ based on the heat diffusion theory (Figure 1). The element distribution from the core to the outside surface can be adjusted by regulating the heating rate and manipulating the diffusion coefficients of the different elements. The gradient distribution of $\mathrm{Zn}$ forms also a gradient of energy levels within the multishell layer, which facilitates the separation and transfer of photogenerated carriers. In addition, the new multishell photocatalyst exhibits stronger light absorption and a higher specific surface area. As a result, a high photocatalytic hydrogen evolution rate of $8001 \mu \mathrm{mol}$ g-1 h-1 has been achieved.

\section{Results and Discussion}

The multishell ZnS-CoS2 microspheres have been prepared by a two-step method (details see Figure 1 and the Experimental Section). First, the $\mathrm{Zn}-$ Co coordination polymer microspheres are produced by a hydrothermal method (Figure S1, Supporting Information), and their amorphous nature is revealed by $\mathrm{X}$-ray diffraction (XRD) (Figure S2, Supporting Information). Then, the $\mathrm{Zn}-\mathrm{Co}$ coordination polymer microspheres are calcined in a muffle furnace under air at $500^{\circ} \mathrm{C}$ with heating rates 1,4 , and ${ }^{\circ} \mathrm{C}$ min-1, to obtain slow-heating multishell $\mathrm{ZnCo}_{2} \mathrm{O}_{4}$ (termed as $\mathrm{S}-\mathrm{ZnCoO}$ ), medium-heating multishell $\mathrm{ZnCo}_{2} \mathrm{O}_{4}$ (termed as $\mathrm{M}-\mathrm{ZnCoO}$ ), and fast-heating multishell $\mathrm{ZnCo}_{2} \mathrm{O}_{4}$ (termed as $\mathrm{F}-\mathrm{ZnCoO}$ ), respectively. The morphology, element distribution, crystal structure of the multishell $\mathrm{ZnCo}_{2} \mathrm{O}_{4}$ have been confirmed as shown in Figures S3-S7 (Supporting Information). In all three multishell materials, a distribution gradient of $\mathrm{Zn}$ from the core to the outermost layer is achieved, this being more prominent in the fast-heating sample, F-ZnCoO (Figure S4, Supporting Information). The multishell ZnS/CoS2 microspheres are obtained by vulcanizing the multishell $\mathrm{ZnCo} 2 \mathrm{O} 4$ microspheres, $\mathrm{S}-\mathrm{ZnCoO}, \mathrm{M}-\mathrm{ZnCoO}$, and $\mathrm{F}-\mathrm{ZnCoO}$, with sulfur powder in a tubular furnace under argon atmosphere. The corresponding multishell $\mathrm{ZnS} / \mathrm{CoS}_{2}$ microspheres are named as S-ZnS/CoS2, M-ZnS/ CoS2, and F-ZnS/CoS2. During the vulcanization process, the gradient distribution of $\mathrm{Zn}$ remains unaltered in all samples.

Figure 2; and Figure S8 (Supporting Information) show the transmission electron microscope (TEM) and scanning electron microscopy (SEM) images of the obtained multishell $\mathrm{ZnS} / \mathrm{CoS} 2$ microspheres. The size of the microspheres is about $2 \mu \mathrm{m}$, similar with that of the multishell $\mathrm{ZnCo} 2 \mathrm{O}_{4}$. The TEM images confirm that the $\mathrm{ZnS} / \mathrm{CoS}_{2}$ microspheres exhibit a multishell structure with 5-7 layers per microsphere. The HRTEM images show lattice fringes with a $d$-spacing of $0.278 \mathrm{~nm}$, attributed to the (200) lattice plane of CoS2 and a lattice spacing of $0.312 \mathrm{~nm}$ assigned to the (001) plane of $\mathrm{ZnS}$ in the $\mathrm{ZnS} / \mathrm{CoS}_{2}$ microspheres.[60,61] The XRD patterns and Raman spectra also confirm the fact that the multishell microspheres are composed of the two sulfides, $\mathrm{ZnS}$ and CoS2 after the sulfidation process of the multishell $\mathrm{ZnCo}_{2} \mathrm{O}_{4}$ microspheres (Figures $\mathrm{S} 9$ and $\mathrm{S} 10$, Supporting Information). Energy-dispersive spectroscopy (EDS) elemental mapping analysis is conducted to estimate the composition distribution of the multishell $\mathrm{ZnS} / \mathrm{CoS}_{2}$ microspheres. As shown in Figure $2 \mathrm{a}-\mathrm{c}$, all three multishell microspheres, $\mathrm{S}-\mathrm{ZnS} / \mathrm{CoS} 2, \mathrm{M}-\mathrm{ZnS} / \mathrm{CoS}_{2}$, and $\mathrm{F}-\mathrm{ZnS} / \mathrm{CoS}_{2}$, are consisted of $\mathrm{Zn}$, Co, and S elements, without the presence of any impurities. In the case of the S-ZnS/CoS2 sample, the three elements $Z n$, Co, and S are uniformly distributed, while multiple shells can be clearly identified. When the heating rate is 
increased to $4{ }^{\circ} \mathrm{C}$ min-1 (corresponding to the $\mathrm{M}-\mathrm{ZnS} / \mathrm{CoS} 2$ sample), both Co and S maintain a uniform distribution, whereas the content of $\mathrm{Zn}$ shows a slight tendency to gradually decrease toward the outer layers of the microspheres. The sample with the fastest heating treatment of $7^{\circ} \mathrm{C}$ min-1 (i.e., F$\mathrm{ZnS} / \mathrm{CoS}_{2}$ ) displays the most obvious gradient distribution of $\mathrm{Zn}$ across all the multishell $\mathrm{ZnS} / \mathrm{CoS}_{2}$ prepared samples. Furthermore, the EDS line scanning profiles demonstrate that the responses of $C_{0}$ and $S$ fluctuate uniformly within the shell structure, proving the formation of a multishell structure. The $\mathrm{Zn}$ content in the $\mathrm{F}-\mathrm{ZnS} / \mathrm{CoS} 2$ sample decreased sharply from the core of the multishell microspheres to the outer layer, consistent with the EDS mapping analysis.

The multishell oxides are formed by the heterogeneous shrinkage during the thermal treatment of $\mathrm{Zn}-$ Co coordination polymer spheres.[18,21,26,30,33,40] The large temperature gradient along the radial direction of the $\mathrm{Zn}-\mathrm{Co}$ coordination polymer sphere results in a transition from an amorphous $\mathrm{Zn}-\mathrm{Co}$ coordination polymer sphere to the oxide layer on the exterior of the $\mathrm{Zn}-\mathrm{Co}$ coordination polymer sphere. As the heating continues, the shrinking process progresses, and eventually multishell oxide particles are formed. The thermal diffusion during the solidification process has a significant effect on the chemical composition distribution.[25,62,63] According to the diffusion formula

$D=D_{0} \exp (-Q / R T)$

where $D$ is the diffusion coefficient, $D 0$ is the frequency factor, $Q$ is the diffusion activation energy, $R$ is the gas constant, and $T$ is the heat treatment temperature.[64] The heat treatment temperature and the diffusion activation energy substantially determine the value of the diffusion coefficient. On one hand, the higher temperature, the larger the diffusion coefficient values. In our current work, for the sample calcined using the fastest heating rate, it means that a higher heat treatment temperature is applied within the same heating time. Thus, a quicker diffusion process takes place. On the other hand, the larger diffusion activation energy, the smaller the diffusion coefficient. The high melting point of the elements in the compound indicates a strong bonding between atoms, meaning that a larger diffusion activation energy is required, and a smaller diffusion coefficient value is obtained. The melting point of $\mathrm{Co}$ is $1495^{\circ} \mathrm{C}$ higher than that of $\mathrm{Zn}\left(420^{\circ} \mathrm{C}\right)$, so the diffusion coefficient of $\mathrm{Zn}$ is higher than that of $\mathrm{Co}$ at the same temperature. Particularly, a higher heating rate (e.g., $7^{\circ} \mathrm{C}$ min-1) accelerates the difference between the diffusion coefficients of $\mathrm{Zn}$ and Co elements. As a result, an obvious gradient distribution of $\mathrm{Zn}$ is created in the multishell $\mathrm{ZnCo} 2 \mathrm{O}_{4}$ microspheres.

To understand the effect of the gradient distribution of $Z n$ on the internal energy band structure of the multishell ZnS/ CoS2 samples, we perform the ultraviolet photoelectron spectra (UPS) in depth by $\mathrm{Ar}_{+}$ etching. Figure $3 a$ illustrates that the $A r+$ etching process from the surface $(0 \mu \mathrm{m})$ to the core of the $\mathrm{F}$ $\mathrm{ZnS} / \mathrm{CoS} 2$ sample $(0.8 \mu \mathrm{m})$. We collect the UPS depth profile spectra of the sample when the etching depth are $0.2,0.4,0.6$, and $0.8 \mu \mathrm{m}$, respectively (Figure S11, Supporting Information). The cut-off regions of UPS spectra at different etch depths are shown in Figure $3 \mathrm{~b}$. The $E_{\text {cutoff }}\left(E_{\text {cutoff }}\right.$ is the energy at which the secondary photoemission begins) of the $\mathrm{F}-\mathrm{ZnS} / \mathrm{CoS} 2$ sample before etching is $16.9 \mathrm{~V}$. After 
the $\mathrm{Ar}$ + beam etching, the Ecutoff at the etching depths of $0.2,0.4,0.6$, and $0.8 \mu \mathrm{m}$ are $17.5,18.3,18.9$, and $19.1 \mathrm{eV}$, respectively. The position of the Fermi level $(E \mathrm{f})$ is determined by formula (2)[65]

$E_{\mathfrak{f}}=21.22 \mathrm{eV}-E_{\text {cutoff }}$

The calculated $E_{\mathrm{f}}$ is $4.32,3.95,3.69,3.41$, and $3.17 \mathrm{eV}$, relative to (the vacuum level) Evac, at different etching depths from surface to core of the $\mathrm{F}-\mathrm{ZnS} / \mathrm{CoS}_{2}$ sample, respectively. The energy band structure of each shell is the overall performance of the energy level positions of individual $\mathrm{ZnS}$ and CoS2 in the shells. The results show that the Fermi level gradually decreases from the inner core to the surface of the multishell F-ZnS/CoS2 sample (Figure 3c). This indicates that the gradient distribution of Zn helps to form a gradient Fermi level inside the multishell. X-ray photoelectron spectroscopy (XPS) has been performed to analyze the surface chemical composition and electronic states of the multishell ZnS/CoS2 samples (Figure S12, Supporting Information). The Zn $2 p$ peak becomes weaker and the binding energy shifts negatively as the heating rate increases, indicating that the $\mathrm{Zn}$ content on the surface of the sample decreases with increasing heating rate,[52,65] which is consistent with the EDS and UPS results.

We also perform transient surface photovoltage (SPV) studies to illustrate the carriers dynamic process of the multishell sulfide samples. This technique can directly demonstrate generation, separation, and recombination behavior of the photogenerated carriers.[1,66-69] The photovoltage intensity is proportional to the excitons separation efficiency: a weaker photovoltage signal indicates a lower carrier separation efficiency.[67,70] The transient SPV spectra of the prepared photocatalysts (Figure 4a) show a negative response peak. Photogenerated electrons accumulate on the sample surface and induce a photocharge separation process, which is conducive to the photocatalytic hydrogen production, taking place at the surface of the material. Interestingly, the F-ZnS/CoS2 sample shows the strongest photovoltage signal compared to the $\mathrm{M}-\mathrm{ZnS} / \mathrm{CoS} 2$ and the $\mathrm{S}-\mathrm{ZnS} / \mathrm{CoS} 2$ samples. This suggests that the dissociation efficiency for photoinduced carriers is effectively promoted through constructing gradient energy levels inside the $\mathrm{F}-\mathrm{ZnS} / \mathrm{CoS}_{2}$ sample. The charge separation efficiency is calculated to be $71.5 \%$ for the F-ZnS/CoS2 sample (see the detailed discussion in the Supporting Information).

To further understand the electrical effects, we perform photoluminescence (PL) analysis and electrochemical characterization, which is commonly used to analysis electron transfer and recombination process at the surface of the photocatalysts. As shown in Figure 4b, PL intensity of the $\mathrm{F}-\mathrm{ZnS} / \mathrm{CoS} 2$ microspheres is the lowest among the three multishell $\mathrm{ZnS} / \mathrm{CoS} 2$ samples, indicating that the gradient energy level in multishell photocatalyst is favorable for charge transfer, thereby reducing carriers recombination. Figure $4 \mathrm{c}$ shows the photocurrent density-time $(I-t)$ curves of the $\mathrm{F}-\mathrm{ZnS} / \mathrm{CoS} 2$, $\mathrm{M}-\mathrm{ZnS} / \mathrm{CoS} 2$, and S-ZnS/CoS2 samples in an on-and-off cycle mode under dark and visible-light conditions. The photocurrent of the F-ZnS/CoS2 sample is $11.86 \mathrm{~mA} \mathrm{~cm}-2$, which is 1.7 and 3.6 times higher than that of the $\mathrm{M}-\mathrm{ZnS} / \mathrm{CoS}_{2}$ and $\mathrm{S}-\mathrm{ZnS} / \mathrm{CoS}_{2}$ samples, respectively. This is due to the more efficient photoexcited electron generation and transfer properties of the F-ZnS/ CoS2 sample. After four switch-on/off cycles, no decrease in the photocurrent density is observed for the three samples, which 
indicates that all photocatalysts possess an excellent recycling stability. The electrochemical impedance spectroscopy data show that $\mathrm{F}-\mathrm{ZnS} / \mathrm{CoS} 2$ exhibits the smallest arc radius, indicating that the sample possesses the fastest interface electron transfer ability among the three materials (Figure S13, Supporting Information).

We also study the absorption spectra of the samples (Figure S14, Supporting Information). Compared with the $\mathrm{M}-\mathrm{ZnS} / \mathrm{CoS} 2$ and $\mathrm{S}-\mathrm{ZnS} / \mathrm{CoS} 2$, the $\mathrm{F}-\mathrm{ZnS} / \mathrm{CoS} 2$ shows the widest and strongest absorption region (extended from 200 to $510 \mathrm{~nm}$ ). In addition, the three multishell $\mathrm{ZnS} / \mathrm{CoS} 2$ samples show stronger absorption capacity than the single nonshell $\mathrm{ZnS}$, or $\operatorname{CoS} 2$ particles, or the mixed $\mathrm{ZnS} / \mathrm{CoS} 2$ particles (see morphology in Figure S15, Supporting Information). All optical and electrical characterizations indicate that the $\mathrm{F}-\mathrm{ZnS} / \mathrm{CoS}_{2}$ sample possesses the best photoelectrical properties among the multishell $\mathrm{ZnS} / \mathrm{CoS}_{2}$ samples. The quantum efficiency of the photohydrogen conversion is a key parameter for evaluating the photocatalytic reaction, therefore we test the external quantum efficiency (EQE) spectra of the S-ZnS/CoS2, M-ZnS/CoS2, and F-ZnS/CoS2 samples under monochromatic illumination from a $100 \mathrm{~mW} \mathrm{~cm}-2$ Xe lamp. As shown in Figure 4d, the F-ZnS/CoS2 photocatalyst shows good EQE in the range of $200-510 \mathrm{~nm}$ and the maximum EQE at $290 \mathrm{~nm}$ is about $54.6 \%$, while the maximum EQE shown in $\mathrm{M}-\mathrm{ZnS} / \mathrm{CoS}_{2}$ and $\mathrm{S}-\mathrm{ZnS} / \mathrm{CoS}_{2}$ are $39.2 \%$ and $21.2 \%$, respectively. EQE results indicate that the $\mathrm{F}-\mathrm{ZnS} / \mathrm{CoS} 2$ photocatalyst has the best photoelectric conversion efficiency, in agreement with the light absorption and photocurrent measurements. In addition, the specific surface areas of the three multishell ZnS/ CoS2 samples have been measured and only slightly differences are observed (Figure S16a,b, Supporting Information). Compared with nonshell ZnS and CoS2 samples (Figure S16c,d, Supporting Information), the multishell $\mathrm{ZnS} / \mathrm{CoS} 2$ samples possess a larger specific surface area, which proves that the multishell structure is more conducive to photocatalysis.

We evaluate the photocatalytic activity of the sample in aqueous solution using a $\mathrm{Na} 2 \mathrm{~S}-\mathrm{Na}_{2} \mathrm{SO}_{3}$ as the hole sacrificial agent under a $100 \mathrm{~mW} \mathrm{~cm}-2$ Xe arc lamp simulating AM 1.5 irradiation. The photocatalytic reaction is performed for four cycles and each cycle lasted $160 \mathrm{~min}$. As shown in Figure $5 \mathrm{a}$, the yield of hydrogen evolution for $\mathrm{S}-\mathrm{ZnS} / \mathrm{CoS}_{2}$ is ranged from $6047 \mu \mathrm{mol} \mathrm{g-1}$ in the first circle to $5600 \mu \mathrm{mol} \mathrm{g-1}$ during the fourth cycle. The yield of hydrogen evolution for M-ZnS/CoS2 is $13252 \mu \mathrm{mol}$ g-1 at the first circle, which is about 2.2 times than that of the S-ZnS/CoS2. Meanwhile, the yield of hydrogen evolution for the $\mathrm{F}-\mathrm{ZnS} / \mathrm{CoS}_{2}$ is $21239 \mu \mathrm{mol} \mathrm{g}-1$ at the first circle which is about 3.5 times higher than that of $\mathrm{S}-\mathrm{ZnS} / \mathrm{CoS}_{2}$. In addition, no significant deactivation is observed in four consecutive cycles, which indicate high stability of the photocatalyst. The calculated hydrogen production rate is shown in Figure 5b. The F-ZnS/CoS2 shows the highest hydrogen production rate of $8001 \mu \mathrm{mol} g-1 \mathrm{~h}-1$, which is $\approx 1.6$ times and 3.5 times than that of the $\mathrm{M}-\mathrm{ZnS} / \mathrm{CoS} 2$ and $\mathrm{S}-\mathrm{ZnS} / \mathrm{CoS} 2$. To corroborate these results, we also investigated the hydrogen production rate of the single $\mathrm{ZnS}, \mathrm{CoS} 2$ materials and their mixture. The hydrogen production rate of the single $\mathrm{ZnS}$ spheres is $522 \mu \mathrm{mol} \mathrm{g-1} \mathrm{h-1.} \mathrm{The} \mathrm{single} \mathrm{CoS2}$ spheres show a low hydrogen production rate because of its metallic feature.[71] After mixing ZnS and CoS2, the hydrogen production rate reaches $710 \mu \mathrm{mol} \mathrm{g-1} \mathrm{h-1} \mathrm{(Figure} \mathrm{S17,} \mathrm{Supporting} \mathrm{Information).}$ The hydrogen production rate of the $\mathrm{F}-\mathrm{ZnS} / \mathrm{CoS}_{2}$ is 15.3 folds and 11.3 folds higher than that of the $\mathrm{ZnS}$ spheres and mixture of $\mathrm{ZnS}$ and $\mathrm{CoS} 2$, which proves that the multishell F-ZnS/CoS2 with gradient 
energy level, stronger light absorption, and larger specific surface area can greatly improve the hydrogen production performance of photocatalysts.

Considering these results, we propose a possible mechanism to explain the enhanced performance of photocatalytic hydrogen production using the $\mathrm{F}-\mathrm{ZnS} / \mathrm{CoS} 2$ system, as shown in Figure $5 \mathrm{c}$. First, the F$\mathrm{ZnS} / \mathrm{CoS} 2$ photocatalyst possesses the ability to absorb more light owing to their unique multishell structure and large specific surface area. Under illumination, the electrons are transferred from the semiconductor ZnS to CoS2 which is in close contact with ZnS (forming a Schottky junction), hence the photogenerated electrons and holes are rapidly separated. Meanwhile, as a gradient Fermi level is created in the F-ZnS/CoS2, electrons are transferred from the higher Fermi level to lower Fermi level. In other words, electrons transfer smoothly from the core to the outside of the multishell F-ZnS/ CoS2, reducing the carrier recombination probability. Finally, the transferred electrons on the photocatalyst surface participate in hydrogen production reaction to improve photocatalytic hydrogen production performance and the photogenerated holes could be consumed by the sacrificial agent.

\section{Conclusion}

In summary, multishell $\mathrm{ZnS} / \mathrm{CoS}_{2}$ microspheres with gradient distribution of $\mathrm{Zn}$ have been designed based on the thermal diffusion theory for the first time. Due to their unique multishell structure, a gradient Fermi level is created from the core to the outside layer, which promotes an efficient carrier separation and electron transfer. In addition, these new multishell photocatalysts exhibit a strong light absorption and large specific surface area. As a result, they show a remarkable performance for hydrogen production reaction, with a hydrogen evolution rate of $8001 \mu \mathrm{mol} \mathrm{g-1} \mathrm{h-1,} \mathrm{which} \mathrm{is} 3.5$ times higher than that of the multishell $\mathrm{ZnS} / \mathrm{CoS} 2$ particles without a gradient distribution of $\mathrm{Zn}$ and 11.3 holds higher than that of the mixture of nonshell $\mathrm{ZnS}$ and $\mathrm{CoS} 2$ nanoparticles. Our work demonstrates that controlling the diffusion rate of the elements present in a semiconductor is an effective route to simultaneously regulate morphology and structure to design efficient photocatalysts.

\section{Experimental Section}

Synthesis of Photocatalysts-Synthesis of the Multishell $\mathrm{ZnCo}_{2} \mathrm{O}_{4}$ Microspheres: The multishell $\mathrm{ZnCo}_{2} \mathrm{O}_{4}$ microspheres were prepared by a hydrothermal method according to the previous report. [18,44] First, $0.033 \mathrm{mmol}$ of hydrated zinc nitrates $\mathrm{Zn}\left(\mathrm{NO}_{3}\right)_{2} \cdot 6 \mathrm{H}_{2} \mathrm{O}, 0.067 \mathrm{mmol}$ of hydrated cobalt nitrates $\mathrm{Co}\left(\mathrm{NO}_{3}\right)_{2} \cdot 6 \mathrm{H}_{2} \mathrm{O}$, and $0.100 \mathrm{mmol}$ of isophthalic acid ( $\left.\mathrm{H}_{2} \mathrm{IPA}\right)$ were dissolved in a mixture of acetone $(5 \mathrm{~mL})$ and DMF $(5 \mathrm{~mL})$, then stirred vigorously for $6 \mathrm{~h}$ to form a homogeneous solution. Subsequently the homogeneous solution was transferred into a $50 \mathrm{~mL}$ stainless-steel autoclave lined Teflon and reacted at $160^{\circ} \mathrm{C}$ for $4 \mathrm{~h}$. After cooling to room temperature, the obtained product was separated by centrifugation, and washed three times with ethanol and distilled water, then dried in a vacuum oven at $80^{\circ} \mathrm{C}$ keep $12 \mathrm{~h}$ to form $\mathrm{Zn}$-Co coordination polymer spheres. Finally, the dried samples were annealed at $500^{\circ} \mathrm{C}$ for $10 \mathrm{~min}$ in a muffle furnace under air with heating rates of 1,4 , and $7^{\circ} \mathrm{C}$ min- 1 to obtain S$\mathrm{ZnCoO}, \mathrm{M}-\mathrm{ZnCoO}$, and $\mathrm{F}-\mathrm{ZnCoO}$, respectively.

Synthesis of Photocatalysts-Synthesis of Multishell ZnS/CoS2 Microspheres: The three multishell ZnS/CoS2 microspheres were synthesized through vulcanizing the as-prepared multishell $\mathrm{ZnCo2O}_{4}$ microspheres in a dual zone tube furnace. The multishell $\mathrm{ZnCo}_{2} \mathrm{O}_{4}$ microspheres were placed in the high temperature zone of the tube furnace; and sulfur powder was placed in the low temperature zone. The residual oxygen in the furnace was removed by applying a pressure of $250 \mathrm{~Pa}$ for $30 \mathrm{~min}$. After that, both low and high temperature zones were heated 
from room temperature to $260^{\circ} \mathrm{C}$ (heating rate $4^{\circ} \mathrm{C}$ min-1) and $500{ }^{\circ} \mathrm{C}$ (heating rate $8{ }^{\circ} \mathrm{C}$ min-1), respectively. The temperature was kept for $1 \mathrm{~h}$ under $\mathrm{Ar}$ gas flow rate of 100 s.c.c.m, and subsequently cooled down to ambient temperature under $\mathrm{Ar}$ atmosphere. The three multishell oxides of $\mathrm{S}-\mathrm{ZnCoO}, \mathrm{M}-\mathrm{ZnCoO}$, and F-ZnCoO were sulfurized following the vulcanization conditions described above to obtain three multishell bisulfide of S-ZnS/CoS2, $\mathrm{M}-\mathrm{ZnS} / \mathrm{CoS} 2$, and F-ZnS/CoS2, respectively.

Synthesis of Photocatalysts-Synthesis of the ZnS Microspheres: The ZnS microspheres were synthesized by a hydrothermal method as previously reported.[72] $0.5 \mathrm{~g} \mathrm{ZnCl} 2$ and $0.3 \mathrm{~g}$ thiourea were dissolved in $20 \mathrm{~mL}$ of deionized water and stirred for $30 \mathrm{~min}$, then transferred into a $50 \mathrm{~mL}$ autoclave and reacted at $160{ }^{\circ} \mathrm{C}$ for $3 \mathrm{~h}$. The white powder product was collected and washed twice with deionized water and ethanol. The resulting product was dried overnight at $60^{\circ} \mathrm{C}$ to obtain the monomer $\mathrm{ZnS}$.

Synthesis of Photocatalysts-Synthesis of the CoS2 Microspheres: The CoS2 microspheres were synthesized by a hydrothermal method as previously reported.[73] $0.549 \mathrm{~g} \mathrm{CoCl} 2$ and $0.62 \mathrm{~g} \mathrm{Na}_{2} \mathrm{~S}_{2} \mathrm{O}_{3} .5 \mathrm{H}_{2} \mathrm{O}$ were dissolved in 60 $\mathrm{mL}$ of deionized water, and stirred vigorously for $0.5 \mathrm{~h}$ to obtain a homogenous solution. The solution was then transferred into a $100 \mathrm{~mL}$ autoclave and maintained at $180^{\circ} \mathrm{C}$ for $12 \mathrm{~h}$. The black products were washed with absolute ethanol and deionized water and dried at $60^{\circ} \mathrm{C}$ to obtain $\mathrm{CoS} 2$ power.

Characterization: The morphologic characterization and EDS analyses were conducted via a field emission SEM (FEI NOVA 450) and TEM (FEI Tecnai F30G2) using a $200 \mathrm{kV}$ accelerating voltage. X-ray diffraction patterns were collected using LabX XRD-7000, with scan rate of $5^{\circ}$ min-1. The XPS spectra were collected using an X-ray photoelectron spectroscopy instrument (XPS) (Axis Supra). The UPS measurements were conducted on the Axis Supra XPS, coupled with in situ argon-ion bombardment equipment (Axis Supra), and etching depth of $5 \mathrm{~nm}$ min-1 (silica wafer was used as reference), with the $\mathrm{He} \mathrm{I}(21.22 \mathrm{eV})$ emission line employed for excitation, and data obtained with a bias of $-5 \mathrm{~V}$. Specifically, the first UPS characterization was conducted before the F-ZnS/CoS2 sample was etched. After that, the sample was etched to a depth of $200 \mathrm{~nm}$ (etching speed of $5 \mathrm{~nm}$ min-1). Then, the second UPS characterization was conducted. The etching and UPS characterization have been repeated for another three times until the etched depth is $800 \mathrm{~nm}$. The optical properties were measured using UV-vis (Shimadzu UV 3150) and Raman (Renishaw inVia, $532 \mathrm{~nm}$ excitation laser, spot diameter of $1 \mathrm{~mm}$ ) spectrometers. EQE measurements were performed with photoelectrochemical quantum efficiency test and analysis system (CELQPCE3000). Transient SPV was measured using a self-assembled surface photovoltaic testing equipment based on lock-in amplifier under the $355 \mathrm{~nm}$ laser, which included a xenon lamp light source (CHF-XM500 W), a phaselocked amplifier (SR830-DSP), a monochromator (Omni-5007), modulation fan (SR540), sample cell and computer. The PL emission spectra were obtained by a fluorescence spectrometer (FLS 980) with $400 \mathrm{~nm}$ Xe arc lamp and $5 \mathrm{~nm}$ slit width. Nitrogen adsorption-desorption isotherms and the Brunauer-Emmett-Teller surface areas were collected at $77 \mathrm{~K}$ using Micromeritics ASAP2010 equipment. The photoelectrochemical test was conducted by a Chenhua 760 electrochemical workstation using a three-electrode system, equipped with a $100 \mathrm{~mW} \mathrm{~cm}-2 \mathrm{Xe}$ arc lamp simulating $\mathrm{AM} 1.5$ irradiation. A $0.3 \mathrm{~m} \mathrm{Na} 2 \mathrm{SO}_{3}$ solution was used as electrolyte and the photocatalysts loaded at fluorine-doped tin oxide (FTO) glass acted as the working electrode. The specific preparation process of the working electrode is as follows: $10 \mathrm{mg}$ of photocatalyst powder was uniformly ultrasonically dispersed in a mixed solution containing $495 \mu \mathrm{L}$ of water, $495 \mu \mathrm{L}$ of ethanol, and $10 \mu \mathrm{L}$ of naphthol (which can improve the stability of the working electrode). Then, a spin coating method was used to coat $200 \mu \mathrm{L}$ of the slurry onto the FTO electrode.[8,58] $\mathrm{Ag} / \mathrm{AgCl}$ electrode and Pt plate were used as reference and counter electrodes, respectively.

Photocatalytic Activity: The photocatalytic experiments were carried out by mixing $0.05 \mathrm{~g}$ of prepared photocatalysts with $50 \mathrm{~mL}$ of deionized water in a sealed quartz reactor containing $0.2 \mathrm{~m} \mathrm{Na} 2 \mathrm{SO}_{3}$ and $0.2 \mathrm{~m} \mathrm{Na} 2 \mathrm{~S}$ as hole sacrificial agent. A $100 \mathrm{~mW}$ cm-2 Xe arc lamp simulating AM 1.5 irradiation and equipped with a fan, which can effectively dissipate the excess heat was employed as light source. During the photocatalytic reaction, a gas 
chromatographer (Shimadzu GC-2014c) equipped with a thermal conductivity detector was used to analyze the generated gas.

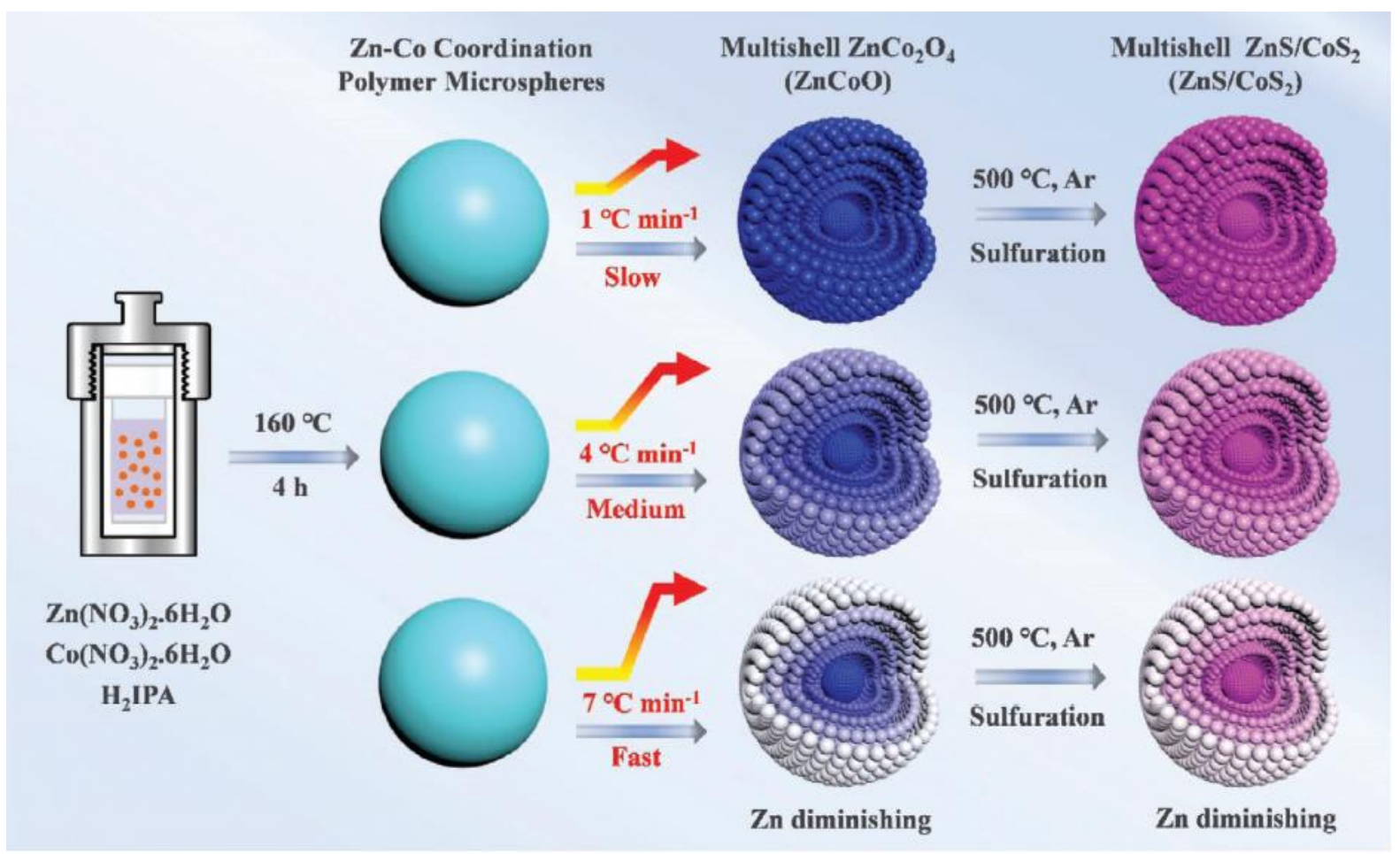

Figure 1. Fabrication process of the S-ZnS/CoS2, M-ZnS/CoS2, and F-ZnS/CoS2. 

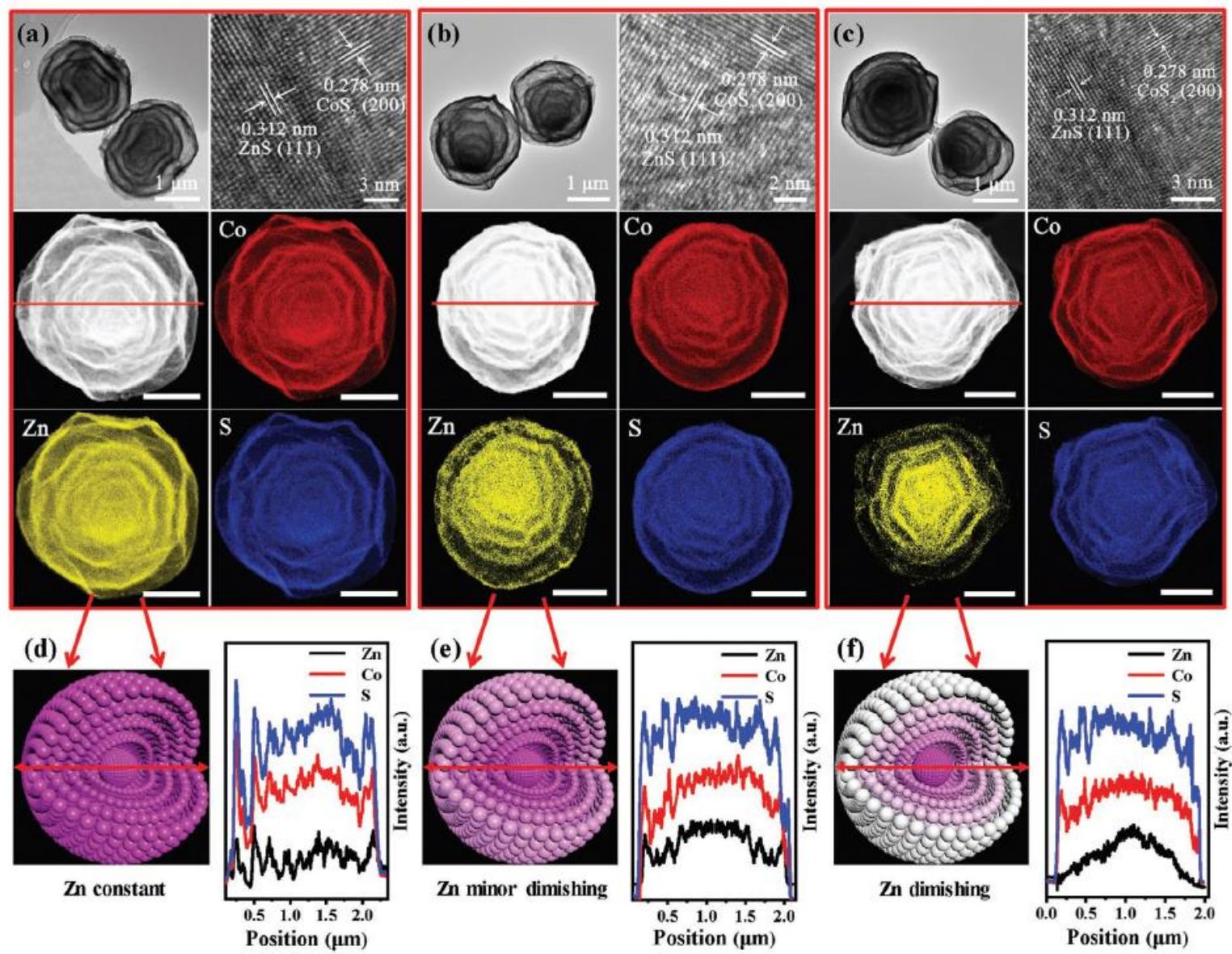

Figure 2. Morphology and microstructure of the a) $\mathrm{S}-\mathrm{ZnS} / \mathrm{CoS}_{2}$, b) $\mathrm{M}-\mathrm{ZnS} / \mathrm{CoS}_{2}$, and c) F-ZnS/CoS2: TEM image, HRTEM image, and EDS element mapping images. Schematic diagram of Zn element distribution and EDS line scan of Zn, Co, and $S$ elements of the d) S-ZnS/CoS2, e) M-ZnS/CoS2, and f) F-ZnS/CoS2. 
(a)

Cross-section

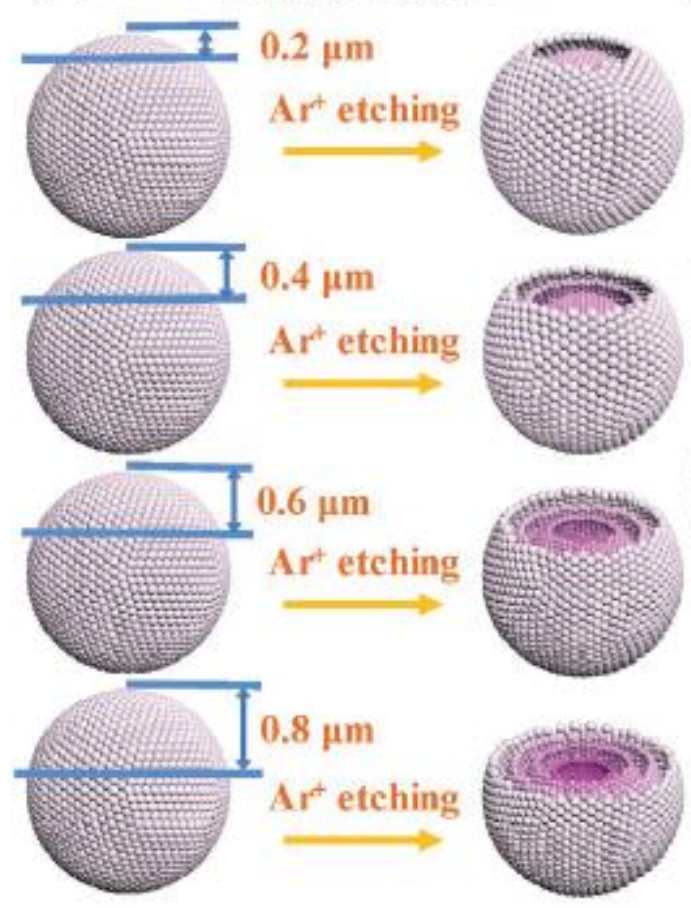

(b)

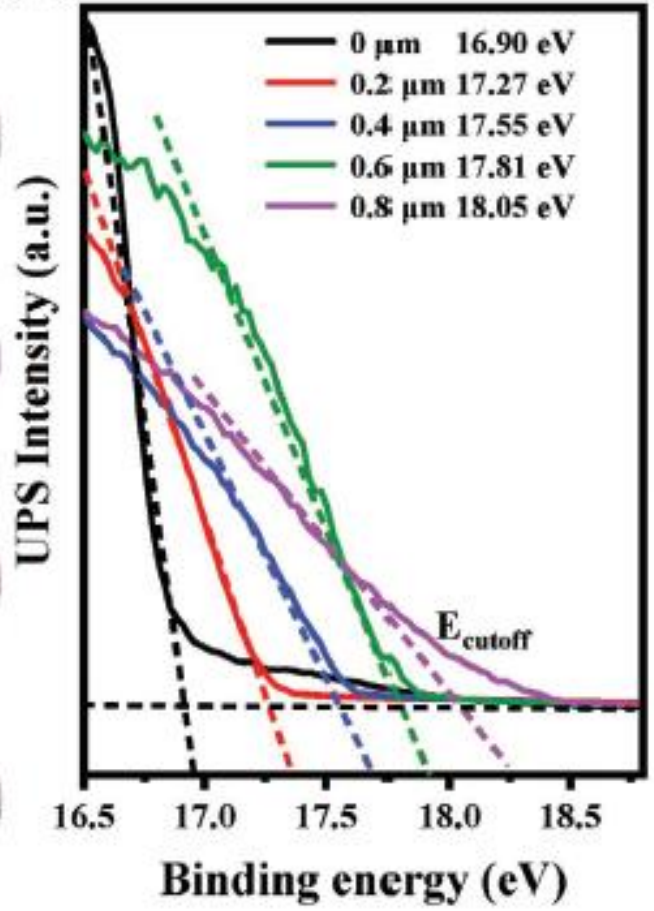

(c)

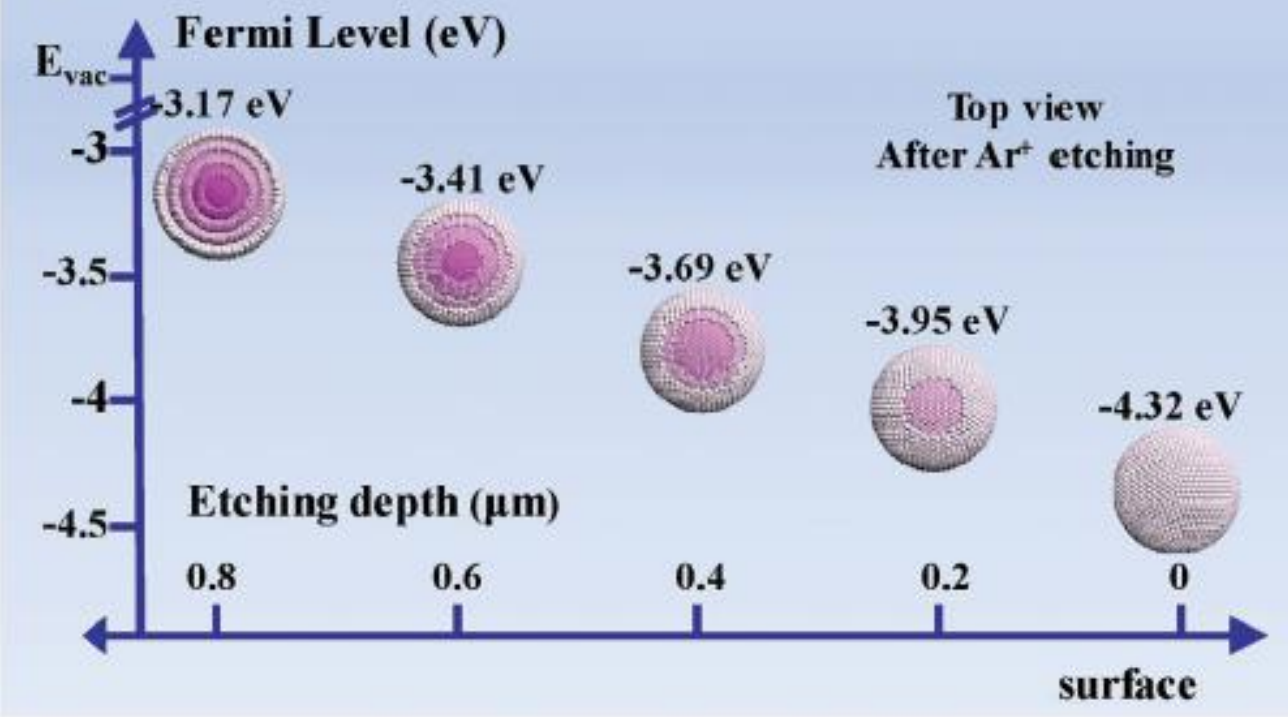

Figure 3. a) UPS etching schematic. b) UPS spectra of the cut-off region for the F-ZnS/CoS2 with different etching depth. c) Fermi energy-level diagram of the F-ZnS/CoS 2 with different etching depth. 
(a)

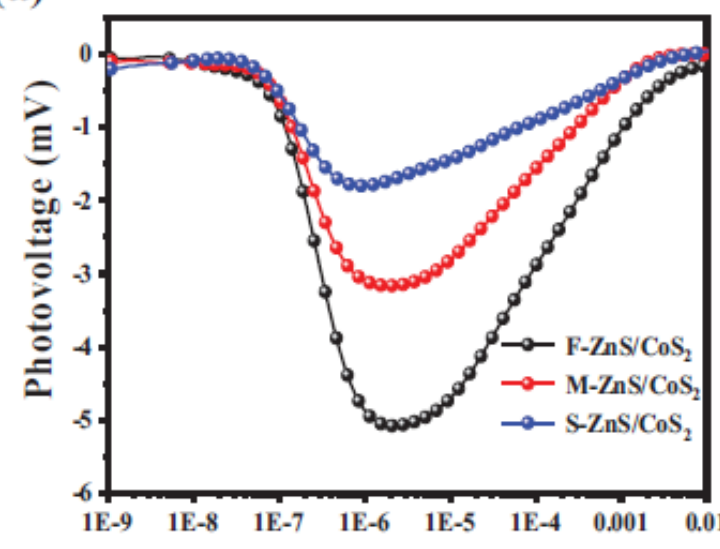

(c)

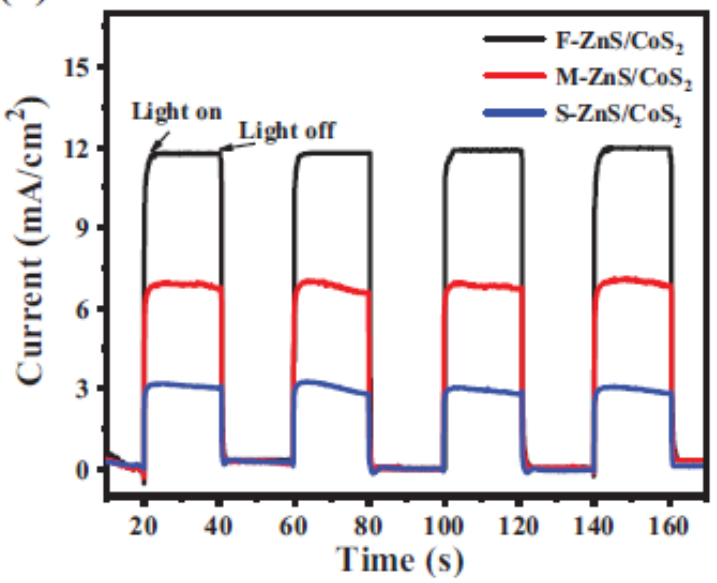

(b)

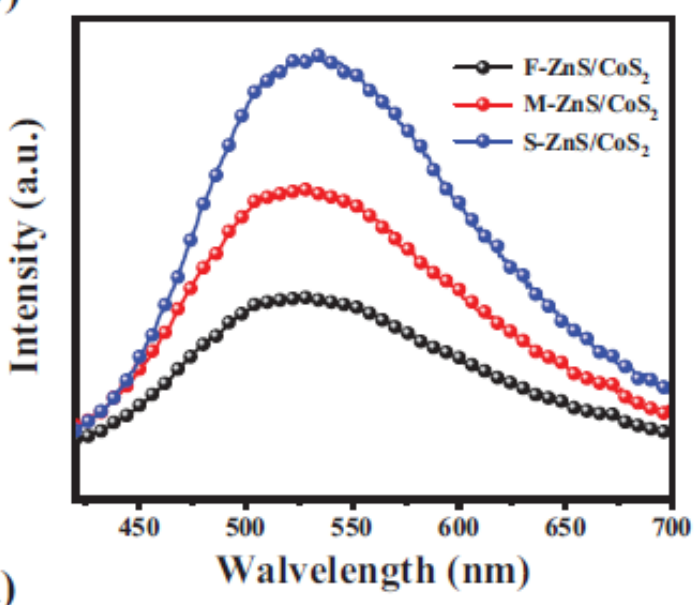

(d)

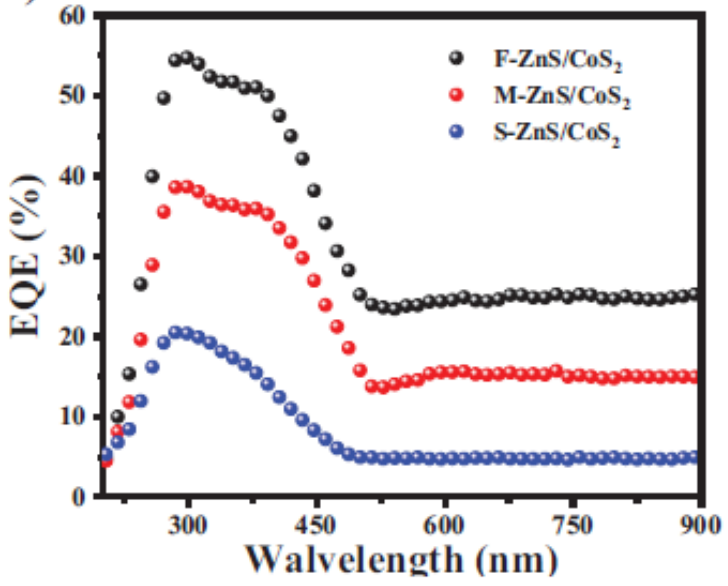

Figure 4. a) Transient surface photovoltage (SPV) spectra of the S-ZnS/CoS2, M-ZnS/CoS2, and F-ZnS/CoS2. b) PL emission spectra of the S-ZnS/CoS2, M-ZnS/CoS2, and F-ZnS/CoS2, excited under $400 \mathrm{~nm}$ Xe light lamp. c) Photocurrent density-time curves of the S-ZnS/CoS2, M-ZnS/CoS2, and F-ZnS/ CoS2. d) EQE spectra of S-ZnS/CoS2, M-ZnS/CoS2, and F-ZnS/CoS2 samples. 
(a)

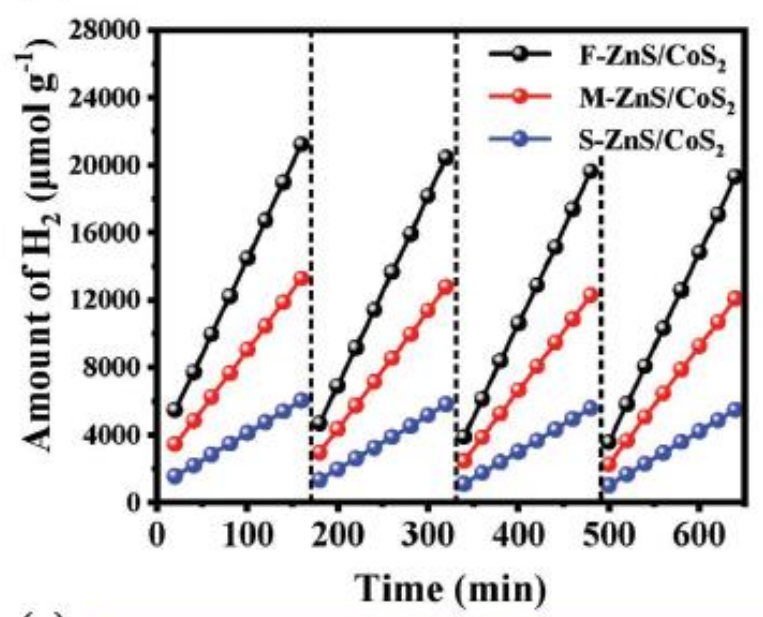

(b)

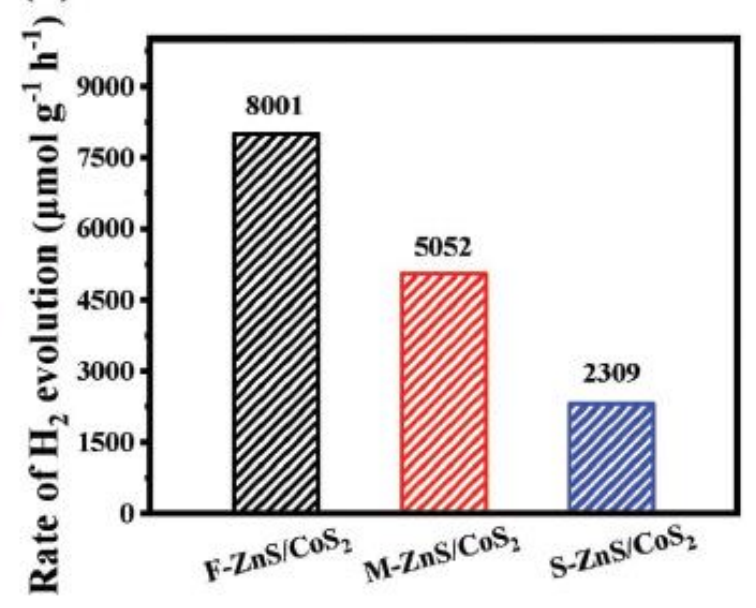

(c)

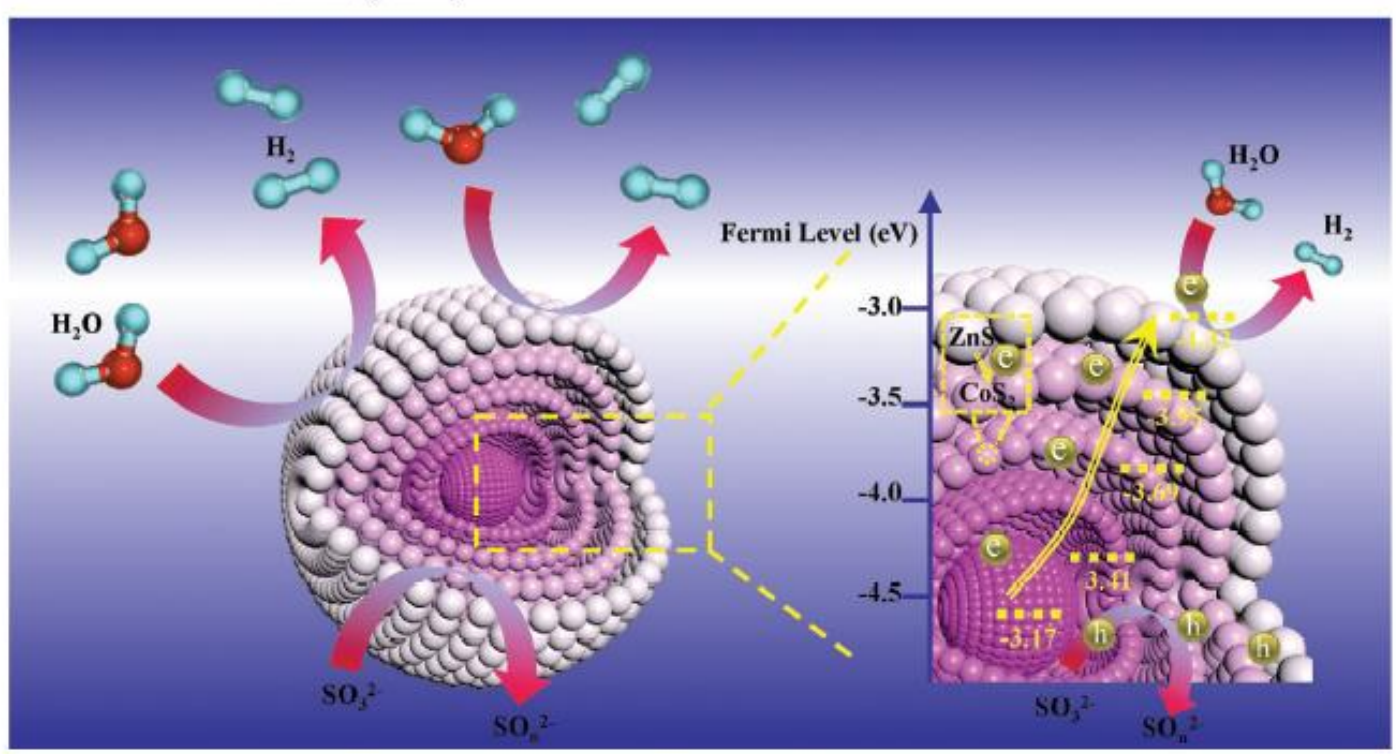

Figure 5. a) Time-dependent photocatalytic hydrogen gas production profiles for the S-ZnS/CoS2, M-ZnS/CoS2, and F-ZnS/CoS2. b) Photocatalytic hydrogen production rate for the S-ZnS/CoS2, M-ZnS/CoS2, and F-ZnS/CoS2. c) Schematic diagram of charge transfer and photocatalytic hydrogen production in the $\mathrm{F}-\mathrm{ZnS} / \mathrm{CoS}_{2}$ under illumination.

\section{Supporting Information}

Supporting Information is available from the Wiley Online Library or from the author.

\section{Acknowledgements}

B.W. and S.G. contributed equally to this work. This research was supported by the Basic Research Fund for Free Exploration in Shenzhen (Grant No. JCYJ20180306171402878), the project of Shaanxi Young Stars in Science and Technology (No. 2017KJXX-18), the Shaanxi International Cooperation Project (No. 2020KWZ-018), and the Fundamental Research Funds for the Central Universities (Nos. 3102019ghxm003, 3102019JC005, and 3102019ghjd001). The authors thank the members from the Analytical \& Testing Center of Northwestern Polytechnical University for the help of SEM, TEM, and UPS characterization.

\section{Conflict of Interest}

The authors declare no conflict of interest.

\section{Keywords}

bisulphides, gradient energy level, heat diffusion, multishell microspheres, photocatalysts 
Received: May 8, 2020

Revised: June 24, 2020

Published online:

[1] J. Ran, J. Qu, H. Zhang, T. Wen, H. Wang, S. Chen, L. Song, X. Zhang, L. Jing, R. Zheng, S. Z. Qiao, Adv. Energy Mater. 2019, 9, 1803402.

[2] M. Sohail, H. Kim, T. W. Kim, Sci. Rep. 2019, 9, 7584.

[3] G. Liao, Y. Gong, L. Zhang, H. Gao, G.-J. Yang, B. Fang, Energy Environ. Sci. 2019, 12, 2080.

[4] Y. Li, Y. K. Peng, L. Hu, J. Zheng, D. Prabhakaran, S. Wu, T. J. Puchtler, M. Li, K. Y. Wong, R. A. Taylor, S. C.

E. Tsang, Nat. Commun. 2019, 10, 4421.

[5] W. Huang, Q. He, Y. Hu, Y. Li, Angew. Chem., Int. Ed. 2019, 131, 8768.

[6] C. Gao, T. Wei, Y. Zhang, X. Song, Y. Huan, H. Liu, M. Zhao, J. Yu, X. Chen, Adv. Mater. 2019, 31, 1806596.

[7] M. Wang, Y. Zuo, J. Wang, Y. Wang, X. Shen, B. Qiu, L. Cai, F. Zhou, S. P. Lau, Y. Chai, Adv. Energy Mater. 2019, 9, 1901801.

[8] S. Guo, X. Li, X. Ren, L. Yang, J. Zhu, B. Wei, Adv. Funct. Mater. 2018, 28, 1802567.

[9] S. Guo, Y. Li, S. Tang, Y. Zhang, X. Li, A. Sobrido, M. Titirici, B. Wei, Adv. Funct. Mater. 2020, https://doi.org/10.1002/adfm.202003035.

[10] L. Buzzetti, G. E. M. Crisenza, P. Melchiorre, Angew. Chem., Int. Ed. 2019, 58, 3730.

[11] Y. Song, X. Xin, S. Guo, Y. Zhang, L. Yang, B. Wang, X. Li, Chem. Eng. J. 2019, 384, 123337.

[12] S. Wang, Y. Wang, S. L. Zhang, S. Q. Zang, X. W. D. Lou, Adv. Mater. 2019, 31, 1903404.

[13] Y. Chao, P. Zhou, N. Li, J. Lai, Y. Yang, Y. Zhang, Y. Tang, W. Yang, Y. Du, D. Su, Y. Tan, S. Guo, Adv. Mater. 2019, 31, 1807226.

[14] Y. Chen, S. Ji, W. Sun, Y. Lei, Q. Wang, A. Li, W. Chen, G. Zhou, Z. Zhang, Y. Wang, L. Zheng, Q. Zhang, L. Gu, X. Han, D. Wang, Y. Li, Angew. Chem., Int. Ed. 2019, 59, 1295.

[15] B. Weng, M.-Y. Qi, C. Han, Z.-R. Tang, Y.-J. Xu, ACS Catal. 2019, 9, 4642.

[16] M.-Q. Yang, C. Han, Y.-J. Xu, J. Phys. Chem. C 2015, 119, 27234.

[17] M. Xiao, Z. Wang, M. Lyu, B. Luo, S. Wang, G. Liu, H. M. Cheng, L. Wang, Adv. Mater. 2019, 31, 1801369.

[18] B. Y. Guan, L. Yu, X. W. Lou, Angew. Chem., Int. Ed. 2017, 56, 2386.

[19] J. Qi, X. Lai, J. Wang, H. Tang, H. Ren, Y. Yang, Q. Jin, L. Zhang, R. Yu, G. Ma, Z. Su, H. Zhao, D. Wang, Chem. Soc. Rev. 2015, 44, 6749.

[20] X. Li, S. Guo, W. Li, X. Ren, J. Su, Q. Song, A. J. Sobrido, B. Wei, Nano Energy 2019, 57, 388.

[21] P. Zhang, X. W. Lou, Adv. Mater. 2019, 31, 1900281.

[22] S. Yu, X. B. Fan, X. Wang, J. Li, Q. Zhang, A. Xia, S. Wei, L. Z. Wu, Y. Zhou, G. R. Patzke, Nat. Commun. 2018, 9, 4009.

[23] Y. T. Liu, D. Li, J. Yu, B. Ding, Angew. Chem., Int. Ed. 2019, 58, 16439.

[24] F. Cao, L. Meng, M. Wang, W. Tian, L. Li, Adv. Mater. 2019, 31, 1806725.

[25] H. Huang, B. Dai, W. Wang, C. Lu, J. Kou, Y. Ni, L. Wang, Z. Xu, Nano Lett. 2017, 17, 3803.

[26] L. Yu, X. Y. Yu, X. W. D. Lou, Adv. Mater. 2018, 30, 1800939.

[27] M. Y. Son, Y. J. Hong, Y. C. Kang, Chem. Commun. 2013, 49, 5678.

[28] F. You, J. Wan, J. Qi, D. Mao, N. Yang, Q. Zhang, L. Gu, D. Wang, Angew. Chem., Int. Ed. 2020, $59,721$.

[29] Y. Wei, J. Wang, R. Yu, J. Wan, D. Wang, Angew. Chem., Int. Ed. 2018, 131, 1436.

[30] J. Wang, Y. Cui, D. Wang, Adv. Mater. 2019, 31, 1801993.

[31] S. Wang, Y. Wang, S. Q. Zang, X. W. Lou, Small Methods 2020, 4, 1900586.

[32] L. Zhou, Z. Zhuang, H. Zhao, M. Lin, D. Zhao, L. Mai, Adv. Mater. 2017, 29, 1602914.

[33] B. Y. Guan, A. Kushima, L. Yu, S. Li, J. Li, X. W. D. Lou, Adv. Mater. 2017, 29, 1605902.

[34] H. Chen, K. Shen, Y. Tan, Y. Li, ACS Nano 2019, 13, 7800. 
[35] Y. Fang, D. Luan, Y. Chen, S. Gao, X. W. Lou, Angew. Chem., Int. Ed. 2020, 59, 7178.

[36] Y. Lin, Z. Qiu, D. Li, S. Ullah, Y. Hai, H. Xin, W. Liao, B. Yang, H. Fan, J. Xu, C. Zhu, Energy Storage Mater. 2018, 11, 67 .

[37] X. Wang, Y. Chen, Y. Fang, J. Zhang, S. Gao, X. W. D. Lou, Angew. Chem., Int. Ed. 2019, 58, 2675.

[38] Y. Lu, J. Nai, X. W. D. Lou, Angew. Chem., Int. Ed. 2018, 57, 2899.

[39] J. Wang, N. Yang, H. Tang, Z. Dong, Q. Jin, M. Yang, D. Kisailus, H. Zhao, Z. Tang, D. Wang, Angew. Chem., Int. Ed. 2013, 52, 6417.

[40] D. Mao, J. Wan, J. Wang, D. Wang, Adv. Mater. 2019, 31, 1802874.

[41] Z. Dong, X. Lai, J. E. Halpert, N. Yang, L. Yi, J. Zhai, D. Wang, Z. Tang, L. Jiang, Adv. Mater. 2012, $24,1046$. [42] G. Zhang, X. W. Lou, Angew. Chem., Int. Ed. 2014, 53, 9041.

[43] M. Waqas, Y. Wei, D. Mao, J. Qi, Y. Yang, B. Wang, D. Wang, Nano Res. 2017, 10, 3920.

[44] F. Qu, W. Shang, D. Wang, S. Du, T. Thomas, S. Ruan, M. Yang, ACS Appl. Mater. Interfaces 2018, 10, 15314.

[45] H. Sun, X. Xu, Z. Yan, C. Xiang, J. Chen, Chem. Mater 2017, 29, 8539.

[46] J. Zhang, J. Wan, J. Wang, H. Ren, R. Yu, L. Gu, Y. Liu, S. Feng, D. Wang, Angew. Chem., Int. Ed. 2019, 58, 5266.

[47] P. Zhang, X. F. Lu, D. Luan, X. W. D. Lou, Angew. Chem., Int. Ed. 2020, 59, 8128.

[48] J. Li, Y. Long, Y. Liu, L. Zhang, Q. Wang, X. Wang, S. Song, H. Zhang, Angew. Chem., Int. Ed. 2020, 59, 1103.

[49] L. Meng, S. Wang, F. Cao, W. Tian, R. Long, L. Li, Angew. Chem., Int. Ed. 2019, 58, 6761.

[50] F. Wang, W. Septina, A. Chemseddine, F. F. Abdi, D. Friedrich, P. D. Bogdanoff, R. van de Krol, S. D. Tilley, S. P. Berglund, J. Am. Chem. Soc. 2017, 139, 15094.

[51] L. Meng, D. Rao, W. Tian, F. Cao, X. Yan, L. Li, Angew. Chem., Int. Ed. 2018, 130, 17124

[52] Y. Yu, Y. Huang, Y. Yu, Y. Shi, B. Zhang, Nano Energy 2018, 43, 236.

[53] S. Wang, Z. Li, Y. Zhang, X. Liu, J. Han, X. Li, Z. Liu, S. Liu, W. C. H. Choy, Adv. Funct. Mater. 2019, 29, 1900417.

[54] Z. Luo, C. Li, S. Liu, T. Wang, J. Gong, Chem. Sci. 2017, 8, 91.

[55] Y. Yu, W. Yan, X. Wang, P. Li, W. Gao, H. Zou, S. Wu, K. Ding, Adv. Mater. 2018, 30, 1705060.

56] H. Sun, C. Neumann, T. Zhang, M. Loffler, A. Wolf, Y. Hou, A. Turchanin, J. Zhang, X. Feng, Adv. Mater. 2019, 31, 1900961.

[57] Y. Zhang, S. Guo, X. Xin, Y. Song, L. Yang, B. Wang, L. Tan, X. Li, Appl. Surf. Sci. 2020, 504, 144291.

[58] X. Xin, Y. Song, S. Guo, Y. Zhang, B. Wang, J. Yu, X. Li, Appl. Catal., B 2020, 269, 118773.

[59] W. S. Subhani, K. Wang, M. Du, X. Wang, S. Liu, Adv. Energy Mater. 2019, 9, 1803785.

[60] H. Liu, R. Hu, J. Qi, Y. Sui, Y. He, Q. Meng, F. Wei, Y. Ren, Y. Zhao, W. Wei, Adv. Mater. Interfaces 2020, 7, 1901659.

[61] Y. P. Xie, Z. B. Yu, G. Liu, X. L. Ma, H.-M. Cheng, Energy Environ. Sci. 2014, 7, 1895.

[62] C. Jiao, Z. Wang, X. Zhao, H. Wang, J. Wang, R. Yu, D. Wang, Angew. Chem., Int. Ed. 2019, 58, 996.

[63] P. Hou, F. Li, Y. Sun, H. Li, X. Xu, T. Zhai, ACS Appl. Mater. Interfaces 2018, 10, 24508.

[64] P. Liu, X. Guo, H. Huang, Q. Yang, Y. Tong, G. A. Hope, Adv. Mater. 2006, 18, 1873.

[65] L. Meng, D. Rao, W. Tian, F. Cao, X. Yan, L. Li, Angew. Chem., Int. Ed. 2018, 130, 17124

[66] C. Han, R. Zhang, Y. Ye, L. Wang, Z. Ma, F. Su, H. Xie, Y. Zhou, P. K. Wong, L. Ye, J. Mater. Chem. A 2019, 7, 9726.

[67] Y. Sang, X. Cao, G. Dai, L. Wang, Y. Peng, B. Geng, J. Hazard. Mater. 2020, 381, 120942.

[68] L. Wang, Y. Hu, F. Qi, L. Ding, J. Wang, X. Zhang, Q. Liu, L. Liu, H. Sun, P. Qu, ACS Appl. Mater. Interfaces 2020, $12,8157$. 
[69] X. Yue, S. Yi, R. Wang, Z. Zhang, S. Qiu, Nano Energy 2018, 47, 463.

[70] L. Bi, X. Gao, L. Zhang, D. Wang, X. Zou, T. Xie, ChemSusChem 2018, 11, 276.

[71] Y. Guo, L. Gan, C. Shang, E. Wang, J. Wang, Adv. Funct. Mater. 2017, 27, 1602699.

[72] Y. Sun, D. Li, Y. Yang, L. Fan, S. Wu, P. Wang, Y. Song, RSC Adv. 2017, 7, 3907.

[73] J. Zhang, Y. Liu, B. Xia, C. Sun, Y. Liu, P. Liu, D. Gao, Electrochim. Acta 2018, 259, 955. 